\title{
SQUAREFREE REVLEX IDEALS
}

\author{
MARILENA CRUPI AND CARMELA FERRÒ
}

\begin{abstract}
We study the combinatorics of squarefree revlex ideals in a polynomial ring in $n$ variables with coefficients in a field $K$. We focus our attention on the relationship between squarefree revlex ideals and revlex ideals. Also, we analyze the extremal Betti numbers of squarefree revlex ideals.
\end{abstract}

1. Introduction. Let $S=K\left[x_{1}, \ldots, x_{n}\right]$ be a polynomial ring in $n$ variables with coefficients in a field $K$. A special class of monomial ideals of $S$ is the class of squarefree monomial ideals. Such ideals are closely related to simplicial complexes. Let $\Delta$ be a simplicial complex on the vertex set $[n]=\{1, \ldots, n\}$ and $I_{\Delta}$ the ideal of $S$ generated by all squarefree monomials $x_{i_{1}} \cdots x_{i_{r}}, 1 \leq i_{1}<\cdots<i_{r} \leq n$, with $\left\{i_{1}, \ldots, i_{r}\right\} \notin \Delta$. It is well known that, if $I$ is an ideal of $S$ generated by squarefree monomials of degree $\geq 2$, then there exists a unique simplicial complex $\Delta$ on $[n]$ with $I=I_{\Delta}$. The ideal $I_{\Delta}$ is known as the Stanley-Reisner ideal of $\Delta$. Stanley [18] and Reisner [17] were the first researchers who used in a systematic way concepts and techniques from commutative algebra to study simplicial complexes by considering the Hilbert function of Stanley-Reisner rings. Since then the study of squarefree monomial ideals from both the algebraic and combinatorial point of view is one of the most inspiring topics in commutative algebra. Some fundamental results on classes of squarefree monomial ideals can be found in $[\mathbf{2}, \mathbf{5}, \mathbf{1 4}]$ and in the references therein.

In this paper, our attention is devoted to squarefree revlex ideals in the polynomial ring $S=K\left[x_{1}, \ldots, x_{n}\right]$. Deery [12] was the first researcher who examined the revlex ideals in the non-squarefree case. He proved an analogue of Bigatti [4], Hulett [15] and Pardue's [16] result about minimal Betti numbers. Precisely, he showed that revlex

2010 AMS Mathematics subject classification. Primary 13A02, 13D02.

Keywords and phrases. Revlex ideal, shifting operation, extremal Betti number. Received by the editors on June 10, 2013, and in revised form on November 25, 2013. 
ideals have the smallest Betti numbers among all stable ideals with the same Hilbert function. Deery's result was generalized in the case of the exterior algebra by Crupi and Ferrò [6].

The outline of the paper is the following. Section 2 contains preliminary notions and results. In Section 3, we study the behaviour of the class of squarefree revlex ideals via tools from simplicial complex theory. This allows us to use the same arguments as in $[6,7]$ in order to study the combinatorics of squarefree revlex ideals (Proposition 3.2 , Corollary 3.3). In Section 4, we prove the squarefree analogue of Deery's result about minimal Betti numbers (Theorem 4.3). In Section 5, we compare the class of revlex ideals with the class of squarefree revlex ideals (Theorems 5.5 and 5.6) by using the shifting operations introduced in [3]. It is well known that there exists a bijection between squarefree strongly stable ideals and strongly stable ideals [3]. Similarly, we show that there exists an injection between the class of squarefree revlex ideals and the class of revlex ideals (Corollary 5.7). In Section 6, we analyze the extremal Betti numbers of squarefree revlex ideals. We show that a squarefree revlex ideal has a unique extremal Betti number (Proposition 6.4) and determine its value (Remark 6.5). Moreover, given a pair of positive integers $(k, \ell)$ with $k \geq 1, \ell \geq 2$, we state conditions for the existence of a squarefree revlex ideal $I$ with the extremal Betti number $\beta_{k, k+\ell}(I)$ (Proposition 6.6).

2. Preliminaries. Let $K$ be a field and $S=K\left[x_{1}, \ldots, x_{n}\right]$ the polynomial ring in $n$ variables with coefficients in $K$. We begin by setting up notation that will be useful.

For a graded ideal $I=\oplus_{j \geq 0} I_{j}$ of $S$, we denote by $\alpha(I)$ the initial degree of $I$, that is, the minimum $s$ such that $I_{s} \neq 0$. Let $\operatorname{Mon}_{d}(S)$ be the set of all monomials of degree $d$ in $S$ and $\operatorname{Mon}_{d}^{s}(S)$ the set of all squarefree monomials of degree $d$ in $S$. For any subset $T$ of $S$, we denote by $\operatorname{Mon}(T)$ the set of all monomials in $T$ and by $\operatorname{Mon}^{s}(T)$ the set of all squarefree monomials in $T$. For a monomial $1 \neq u \in S$, we set

$$
\operatorname{supp}(u)=\left\{i: x_{i} \text { divides } u\right\},
$$

and we write

$$
\max (u)=\max \{i: i \in \operatorname{supp}(u)\}, \quad \min (u)=\min \{i: i \in \operatorname{supp}(u)\} .
$$

Moreover, we set $\max (1)=\min (1)=0$. 
For a monomial ideal $I \varsubsetneqq S$, we denote by $G(I)$ the unique minimal set of monomial generators of $I$. Let $I \subsetneq S$ be a monomial ideal. $I$ is called stable if, for all $u \in G(I)$, one has $\left(x_{j} u\right) / x_{\max (u)} \in I$ for all $j<\max (u)$. $I$ is called strongly stable if, for all $u \in G(I)$, one has $\left(x_{j} u\right) / x_{i} \in I$ for all $i \in \operatorname{supp}(u)$ and all $j<i$.

Let $>_{\text {revlex }}$ be the usual reverse lexicographic order (revlex order, for short) on the monomials of $S$. A subset $M \subsetneq \operatorname{Mon}_{d}(S)$ is a revlex segment of degree $d$ if, for $u \in M, v \in \operatorname{Mon}_{d}(S)$ such that $v>_{\text {revlex }} u$, then $v \in M$.

Definition 2.1. A monomial ideal $I \subsetneq S$ is called a revlex ideal of $S$ if $\operatorname{Mon}\left(I_{d}\right)$ is a revlex segment of degree $d$ for all $d$.

Let $I \subsetneq S$ be a squarefree monomial ideal. $I$ is squarefree stable if, for all $u \in G(I)$, one has $\left(x_{j} u\right) / x_{\max (u)} \in I$ for all $j<\max (u), j \notin \operatorname{supp}(u)$. $I$ is squarefree strongly stable if, for all $u \in G(I)$, one has $\left(x_{j} u\right) / x_{i} \in I$ for all $i \in \operatorname{supp}(u)$ and all $j<i, j \notin \operatorname{supp}(u)$.

For every $1 \leq d \leq n$, we order $\operatorname{Mon}_{d}^{s}(S)$ with the squarefree reverse lexicographic order (squarefree revlex order, for short) $\geq_{\text {srevlex }}$. Let $u=$ $x_{i_{1}} x_{i_{2}} \cdots x_{i_{d}}$ and $v=x_{j_{1}} x_{j_{2}} \cdots x_{j_{d}}$, with $1 \leq i_{1}<i_{2}<\cdots<i_{d} \leq n$, $1 \leq j_{1}<j_{2}<\cdots<j_{d} \leq n$, be squarefree monomials of degree $d$ in $S$. Then

$$
u>_{\text {srevlex }} v \text { if } i_{d}=j_{d}, i_{d-1}=j_{d-1}, \ldots, i_{s+1}=j_{s+1} \text { and } i_{s}<j_{s},
$$

for some $1 \leq s \leq d$. A nonempty set $M \subsetneq \operatorname{Mon}_{d}^{s}(S)$ is called a squarefree revlex segment of degree $d$ if, for $u \in M, v \in \operatorname{Mon}_{d}^{s}(S)$ such that $v>_{\text {srevlex }} u$. Then $v \in M$.

Definition 2.2. Let $I \subsetneq S$ be a squarefree monomial ideal. $I$ is a squarefree revlex ideal if, for all $1 \leq d \leq n$, if $u, v \in \operatorname{Mon}_{d}^{s}(S)$ with $u \in I$ and $v>_{\text {srevlex }} u$, then $v \in I$.

Equivalently, a graded ideal $I \subsetneq S$ is a squarefree revlex ideal if and only if $\operatorname{Mon}^{s}\left(I_{d}\right)$ is a squarefree revlex segment of degree $d$ for all $1 \leq d \leq n$. 
Now, let $M$ be a subset of monomials of $\operatorname{Mon}_{d}(S)$. The set

$$
\left\{x_{i} u: u \in M, \quad i=1, \ldots, n\right\}
$$

of monomials of degree $d+1$ of $S$ is called the shadow of $M$ and is denoted by $\operatorname{Shad}(M)$.

If $M$ is a set of squarefree monomials of $\operatorname{Mon}_{d}^{s}(S)$, the set

$$
\left\{x_{i} u: u \in M, i \notin \operatorname{supp}(u), i=1, \ldots, n\right\}
$$

of squarefree monomials of degree $d+1$ of $S$ is called the squarefree shadow of $M$ and is denoted by $\operatorname{Shad}^{s}(M)$.

Remark 2.3. If $M$ is a revlex segment of degree $d$, then $\operatorname{Shad}(M)$ is not in general a revlex segment of degree $d+1$. Let $M=\left\{x_{1}^{2}, x_{1} x_{2}\right\}$ be a revlex segment of degree 2 of $S=K\left[x_{1}, x_{2}, x_{3}\right]$. Then

$$
\operatorname{Shad}(M)=\left\{x_{1}^{3}, x_{1}^{2} x_{2}, x_{1} x_{2}^{2}, x_{1}^{2} x_{3}, x_{1} x_{2} x_{3}\right\}
$$

is not a revlex segment of degree 3 . In fact $x_{2}^{3}>_{\text {revlex }} x_{1}^{2} x_{3}$, but $x_{2}^{3} \notin \operatorname{Shad}(M)$.

Similarly, if $M$ is a squarefree revlex segment of degree $d$, then $\operatorname{Shad}^{s}(M)$ need not be a squarefree revlex segment of degree $d+1$. For example, if $S=K\left[x_{1}, x_{2}, x_{3}, x_{4}, x_{5}\right]$ and $M=\left\{x_{1} x_{2}, x_{1} x_{3}\right\}$, then

$$
\operatorname{Shad}^{s}(M)=\left\{x_{1} x_{2} x_{3}, x_{1} x_{2} x_{4}, x_{1} x_{3} x_{4}, x_{1} x_{2} x_{5}, x_{1} x_{3} x_{5}\right\}
$$

is not a squarefree revlex segment of degree 3 . In fact, $x_{2} x_{3} x_{4}>_{\text {srevlex }}$ $x_{1} x_{2} x_{5}$, but $x_{2} x_{3} x_{4} \notin \operatorname{Shad}^{s}(M)$.

3. Squarefree revlex ideals. In this section, we analyze the class of squarefree revlex ideals in $S=K\left[x_{1}, \ldots, x_{n}\right]$.

The notion of simplicial complex plays a key role in our analysis. A simplicial complex $\Delta$ on the vertex set $[n]=\{1, \ldots, n\}$ is a collection of subsets of $[n]$ such that

(a) $\{i\} \in \Delta$ for every $i \in[n]$, and

(b) $\sigma \in \Delta, \tau \subseteq \sigma \Rightarrow \tau \in \Delta$.

The ideal $I_{\Delta}$ of $S$ generated by all squarefree monomials $x_{i_{1}} \cdots x_{i_{r}}, 1 \leq$ $i_{1}<\cdots<i_{r} \leq n$, with $\left\{i_{1}, \ldots, i_{r}\right\} \notin \Delta$ is called the Stanley-Reisner ideal of $\Delta$ over $K$, and the quotient algebra $K[\Delta]=S / I_{\Delta}$ is called the 
Stanley-Reisner ring of $\Delta$ over $K$. If $J_{\Delta}=\left(I_{\Delta}, x_{1}^{2}, \ldots, x_{n}^{2}\right)$, then the graded $K$-algebra $K\{\Delta\}=S / J_{\Delta}$ is called the indicator algebra of $\Delta$. If $\Gamma$ is the simplicial complex consisting of all the subsets of the vertex set $[n]$, then the indicator algebra of $\Gamma$ is:

$$
K\{\Gamma\}=K\left[x_{1}, \ldots, x_{n}\right] /\left(x_{1}^{2}, \ldots, x_{n}^{2}\right),
$$

and the set of all squarefree monomials of $S$ forms a basis of $K\{\Gamma\}$.

Definition 3.1. A simplicial complex $\Delta$ is called strongly stable (revlex) if the Stanley-Reisner ideal $I_{\Delta}$ is a squarefree strongly stable ideal (squarefree revlex ideal, respectively).

Let $I \subsetneq S$ be a squarefree monomial ideal, and let $I_{\Gamma}$ be the image of $I$ in $K\{\Gamma\}$. The structure of $K\{\Gamma\}$ ensures that $I$ is a squarefree (strongly) stable ideal in $S$ if and only if $I_{\Gamma}$ is a (strongly) stable ideal in $K\{\Gamma\}$, in the sense of the exterior algebra $E[\mathbf{1}, \mathbf{2}]$. Hence, in order to study the behavior of the squarefree revlex ideals in $S$ that are squarefree strongly stable ideals, one can use the same arguments as in $[6,7]$.

Proposition 3.2. Let $M$ be a squarefree revlex segment of degree $d$ of $S$ such that $d<n-2$. The following conditions are equivalent:

(1) $\operatorname{Shad}^{s}(M)$ is a squarefree revlex segment of degree $d+1$;

(2) $x_{n-(d+1)} \cdots x_{n-3} x_{n-2} \in M$.

Proof. Let $M_{\Gamma}$ be the image of $M$ in $K\{\Gamma\}=K\left[x_{1}, \ldots, x_{n}\right] /\left(x_{1}^{2}, \ldots, x_{n}^{2}\right)$. It is clear that $M$ is a squarefree revlex segment in $S$ if and only if $M_{\Gamma}$ is a revlex segment in $K\{\Gamma\}$. Hence, the assertion follows from [6, Corollary 3.8].

Corollary 3.3. Let $M=\left\{u_{1}, \ldots, u_{t}\right\}$ be a set of monomials of $S$, $d_{1}=\min \left\{\operatorname{deg}\left(u_{i}\right): i=1, \ldots, t\right\}$ and $d_{2}=\max \left\{\operatorname{deg}\left(u_{i}\right): i=1, \ldots, t\right\}$ with $d_{2}<n-2$. Then $I=(M)$ is a squarefree revlex ideal if and only if:

(1) $\operatorname{Mon}^{s}\left(I_{d}\right)$ is a squarefree revlex segment for $d_{1} \leq d \leq d_{2}$;

(2) $x_{n-\left(d_{2}+1\right)} \cdots x_{n-3} x_{n-2} \in M$. 
Proof. See [6, Corollary 3.9].

Proposition 3.4. A squarefree revlex ideal $I \subsetneq S$ is minimally generated in at most two consecutive degrees.

Proof. Let $d=\alpha(I)$. Then $x_{1} x_{2} \cdots x_{d} \in \operatorname{Mon}^{s}\left(I_{d}\right)$ and $x_{1} x_{2} \cdots x_{d} x_{n}$ $\in \operatorname{Mon}^{s}\left(I_{d+1}\right)$. As $I$ is a squarefree revlex ideal and $x_{n-(d+1)} \cdots x_{n-2} x_{n-1}$ $>_{\text {srevlex }} x_{1} x_{2} \cdots x_{d} x_{n}$, it follows that $x_{n-(d+1)} \cdots x_{n-2} x_{n-1} \in \operatorname{Mon}^{s}\left(I_{d+1}\right)$. Hence, $x_{n-(d+1)} \cdots x_{n-2} x_{n-1} x_{n}$ is the smallest monomial belonging to $\operatorname{Shad}^{s}\left(\operatorname{Mon}^{s}\left(I_{d+1}\right)\right)$, with respect to $>_{\text {srevlex }}$, and consequently, $\operatorname{Shad}^{s}\left(\operatorname{Mon}^{s}\left(I_{d+1}\right)\right)=\operatorname{Mon}_{d+2}^{s}(S)$. Therefore, the minimal monomial generators of $I$ are at most of degrees $d$ and $d+1$.

4. A lower bound. Aramova, Herzog and Hibi [3, Theorem 2.9] proved that, over a field $K$ of characteristic zero, the graded Betti numbers of a squarefree monomial ideal are bounded by those of the corresponding squarefree lexicographic ideal. In this section, we prove the squarefree analogue of Deery's theorem [12, Theorem 3.10] on the lower bounds of Betti numbers of a given Hilbert function.

If $I \varsubsetneqq S$ is a monomial ideal and $G(I)$ its unique minimal set of monomial generators, we define the following sets:

$$
\begin{aligned}
G(I)_{d} & =\{u \in G(I): \operatorname{deg}(u)=d\}, \\
G(I ; i) & =\{u \in G(I): \max (u)=i\}, \\
\mathrm{m}_{i}(I) & =|G(I ; i)|, \\
\mathrm{m}_{\leq i}(I) & =\sum_{j \leq i} \mathrm{~m}_{j}(I),
\end{aligned}
$$

for $d>0$ and $1 \leq i \leq n$.

Lemma 4.1. Let $J \varsubsetneqq S$ be a squarefree revlex ideal generated in degree $d$, and let $I \varsubsetneqq S$ be a squarefree strongly stable ideal generated in the same degree such that $\left|\operatorname{Mon}^{s}\left(J_{d}\right)\right| \geq\left|\operatorname{Mon}^{s}\left(I_{d}\right)\right|$. Then,

$$
m_{\leq i}(J) \geq m_{\leq i}(I), \text { for } 1 \leq i \leq n
$$

Proof. Let $\widetilde{J}=J_{\Gamma}$ and $\widetilde{I}=I_{\Gamma}$ be the images of $J$ and $I$ in $K\{\Gamma\}$, respectively. It follows that $\left|\operatorname{Mon}^{s}\left(J_{d}\right)\right|=\operatorname{dim}_{K} \widetilde{J}_{d}$ and $\left|\operatorname{Mon}^{s}\left(I_{d}\right)\right|=$ $\operatorname{dim}_{K} \widetilde{I}_{d}$. The assertion now follows from [6, Theorem 5.3]. 
Lemma 4.2. ([2, Lemma 3.7]). Let $I \varsubsetneqq K\{\Gamma\}$ be a strongly stable ideal with all generators in degree $d<n$. Then

$$
\operatorname{dim}_{K}\left(I_{d+1}\right)=\sum_{i=1}^{n-1} \mathrm{~m}_{i}(I)(n-i)=\sum_{i=1}^{n-1} \mathrm{~m}_{\leq i}(I) .
$$

Hence, using the same arguments as in [2, Theorem 4.4] (see also [14, Theorem 7.4.3]) and [6, Theorem 5.6], one can state the following theorem.

Theorem 4.3. Let $\Delta$ be a strongly stable simplicial complex and $\Delta^{\prime}$ a revlex simplicial complex such that $H_{K\{\Delta\}}(t)=H_{K\left\{\Delta^{\prime}\right\}}(t)$ for all $t$. Then

$$
\beta_{i, j}\left(I_{\Delta}\right) \geq \beta_{i, j}\left(I_{\Delta^{\prime}}\right), \quad \text { for all } i \text { and } j
$$

Proof. Set $I=I_{\Delta}$ and $J=I_{\Delta^{\prime}}$. From [14, Corollary 7.4.2], we have:

$$
\beta_{i, i+j}(I)=\sum_{u \in G(I)_{j}}\left(\begin{array}{c}
\max (u)-j \\
i
\end{array}\right), \quad \text { for all } i, j .
$$

Let $I_{\langle d\rangle}$ be the ideal in $K\{\Delta\}$ generated by all (squarefree) monomials in $I$ of degree $d$.

Since $G(I)_{d+1}=G\left(I_{\langle d+1\rangle}\right)-G\left(I_{\langle d\rangle}\right)\left\{x_{1}, \ldots, x_{n}\right\}$, the above sum can be written as a difference $\beta_{i, i+j}(I)=C-D$, with

$$
\begin{aligned}
C & =\sum_{u \in G\left(I_{\langle j\rangle}\right)}\left(\begin{array}{c}
\max (u)-j \\
i
\end{array}\right) \\
& =\sum_{t=1}^{n} \sum_{u \in G\left(I_{\langle j\rangle} ; t\right)}\left(\begin{array}{c}
t-j \\
i
\end{array}\right)=\sum_{t=1}^{n} \mathrm{~m}_{t}\left(I_{\langle j\rangle}\right)\left(\begin{array}{c}
t-j \\
i
\end{array}\right) \\
& =\sum_{t=1}^{n}\left(\mathrm{~m}_{\leq t}\left(I_{\langle j\rangle}\right)-\mathrm{m}_{\leq t-1}\left(I_{\langle j\rangle}\right)\right)\left(\begin{array}{c}
t-j \\
i
\end{array}\right)=\mathrm{m}_{\leq n}\left(I_{\langle j\rangle}\right)\left(\begin{array}{c}
n-j \\
i
\end{array}\right) \\
& +\sum_{t=1}^{n-1} \mathrm{~m}_{\leq t}\left(I_{\langle j\rangle}\right)\left[\left(\begin{array}{c}
t-j \\
i
\end{array}\right)-\left(\begin{array}{c}
(t+1)-j \\
i
\end{array}\right)\right] \\
& =\mathrm{m}_{\leq n}\left(I_{\langle j\rangle}\right)\left(\begin{array}{c}
n-j \\
i
\end{array}\right)-\sum_{t=j}^{n-1} \mathrm{~m}_{\leq t}\left(I_{\langle j\rangle}\right)\left(\begin{array}{c}
t-j \\
i-1
\end{array}\right)
\end{aligned}
$$


and

$$
D=\sum_{u \in G\left(I_{\langle j-1\rangle}\right)\left\{x_{1}, \ldots, x_{n}\right\}}\left(\begin{array}{c}
\max (u)-j \\
i
\end{array}\right)=\sum_{t=j}^{n} m_{\leq t-1}\left(I_{\langle j-1\rangle}\right)\left(\begin{array}{c}
t-j \\
i
\end{array}\right),
$$

from Lemma 4.2. On the other hand, since the number of generators of $I_{\langle d\rangle}$ and $J_{\langle d\rangle}$ are equal for all $d$, we have $\mathrm{m}_{\leq n}\left(I_{\langle d\rangle}\right)=\mathrm{m}_{\leq n}\left(J_{\langle d\rangle}\right)$. Hence, from Lemma $4.1, \mathrm{~m}_{\leq i}\left(J_{\langle d\rangle}\right) \geq \mathrm{m}_{\leq i}\left(I_{\langle d\rangle}\right)$ for $1 \leq i \leq n$, and consequently:

$$
\begin{aligned}
\beta_{i, i+j}(I)= & \mathrm{m}_{\leq n}\left(I_{\langle j\rangle}\right)\left(\begin{array}{c}
n-j \\
i
\end{array}\right)-\sum_{t=j}^{n-1} \mathrm{~m}_{\leq t}\left(I_{\langle j\rangle}\right)\left(\begin{array}{l}
t-j \\
i-1
\end{array}\right) \\
& -\sum_{t=j}^{n} \mathrm{~m}_{\leq t-1}\left(I_{\langle j-1\rangle}\right)\left(\begin{array}{c}
t-j \\
i
\end{array}\right) \\
\geq & \mathrm{m}_{\leq n}\left(J_{\langle j\rangle}\right)\left(\begin{array}{c}
n-j \\
i
\end{array}\right)-\sum_{t=j}^{n-1} \mathrm{~m}_{\leq t}\left(J_{\langle j\rangle}\right)\left(\begin{array}{c}
t-j \\
i-1
\end{array}\right) \\
& -\sum_{t=j}^{n} \mathrm{~m}_{\leq t-1}\left(J_{\langle j-1\rangle}\right)\left(\begin{array}{c}
t-j \\
i
\end{array}\right)=\beta_{i, i+j}(J) .
\end{aligned}
$$

5. Revlex ideals and shifting operations. In this section, we prove that there is a bijection between the revlex ideals generated in two consecutive degrees and squarefree revlex ideals. Let $I \subsetneq S=$ $K\left[x_{1}, \ldots, x_{n}\right]$ be a squarefree stable ideal. In [2, page 361], for every $w \in \operatorname{Mon}^{s}(I)$, there exists a unique pair $(u, v)$ of squarefree monomials in $S$ such that:

$$
u \in G(I), \quad w=u v, \quad \max (u)<\min (v) .
$$

In [3] two shifting operations are introduced.

Let $u=x_{i_{1}} x_{i_{2}} \cdots x_{i_{j}} \cdots x_{i_{d}}$ be a monomial of $S$, with $i_{1} \leq i_{2} \leq$ $\cdots \leq i_{j} \leq \cdots \leq i_{d} \leq n$, and set

$$
u^{\sigma}=x_{i_{1}} x_{i_{2}+1} \cdots x_{i_{j}+(j-1)} \cdots x_{i_{d}+(d-1)} .
$$

It is easy to verify that $\max \left(u^{\sigma}\right)-\operatorname{deg}\left(u^{\sigma}\right)=\max (u)-1$. If $I$ is a monomial ideal of $S$ with $G(I)=\left\{u_{1}, \ldots, u_{s}\right\}$, we denote by $I^{\sigma}$ the squarefree monomial ideal generated by $u_{1}^{\sigma}, \ldots, u_{s}^{\sigma}$ in $K\left[x_{1}, \ldots, x_{m}\right]$, where $m=\max \{\max (u)+\operatorname{deg} u-1: u \in G(I)\}$. 
There is another operation $\tau$ which is inverse to $\sigma$. For a squarefree monomial $u=x_{i_{1}} x_{i_{2}} \cdots x_{i_{j}} \cdots x_{i_{d}}$ of $S$ with $1 \leq i_{1}<\cdots<i_{j}<\cdots<$ $i_{d} \leq n$, we set

$$
u^{\tau}=x_{i_{1}} x_{i_{2}-1} \cdots x_{i_{j}-(j-1)} \cdots x_{i_{d}-(d-1)} .
$$

Note that $\max \left(u^{\tau}\right)=\max (u)-\operatorname{deg} u+1$. If $I \subsetneq S$ is a squarefree monomial ideal with $G(I)=\left\{u_{1}, \ldots, u_{s}\right\}$, then we write $I^{\tau}$ for the monomial ideal generated by $u_{1}^{\tau}, \ldots, u_{s}^{\tau}$ in $K\left[x_{1}, \ldots, x_{m}\right]$, where $m=$ $\max \{\max (u)-\operatorname{deg} u+1: u \in G(I)\}$.

Remark 5.1. It is easy to verify that, if $u, v \in S_{d}$ and $v>_{\text {revlex }} u$, then $v^{\sigma}>_{\text {srevlex }} u^{\sigma}$ in $K\left[x_{1}, \ldots, x_{m}\right], m=\max \{\max (u)+\operatorname{deg} u-1$ : $u \in G(I)\}$.

Moreover, if $u, v \in \operatorname{Mon}_{d}^{s}(S)$ and $v>_{\text {srevlex }} u$, then $v^{\tau}>_{\text {revlex }} u^{\tau}$ in $K\left[x_{1}, \ldots, x_{m}\right], m=\max \{\max (u)-\operatorname{deg} u+1: u \in G(I)\}$.

The next results establish that there is a bijection between strongly stable ideals and squarefree strongly stable ideals in polynomial rings.

Lemma 5.2. ([3, Lemma 1.2]). Let $I \subsetneq S$ be a strongly stable ideal with $G(I)=\left\{u_{1}, \ldots, u_{s}\right\}$. Set $m=\max \{\max (u)+\operatorname{deg} u-1: u \in G(I)\}$. Then $I^{\sigma}$ is a squarefree strongly stable ideal of $K\left[x_{1}, \ldots, x_{m}\right]$ with $G\left(I^{\sigma}\right)=\left\{u_{1}^{\sigma}, \ldots, u_{s}^{\sigma}\right\}$.

Lemma 5.3. ([3, Lemma 1.4]). Let $I \subsetneq S$ be a squarefree strongly stable ideal with $G(I)=\left\{u_{1}, \ldots, u_{s}\right\}$. Set $m=\max \{\max (u)-\operatorname{deg} u+1$ : $u \in G(I)\}$. Then $I^{\tau}$ is a strongly stable ideal of $K\left[x_{1}, \ldots, x_{m}\right]$ with $G\left(I^{\tau}\right)=\left\{u_{1}^{\tau}, \ldots, u_{s}^{\tau}\right\}$.

Remark 5.4. From Lemma 5.2, one deduces that, if $I \subsetneq S$ is a strongly stable ideal generated in degrees $d_{1}<d_{2}<\cdots<d_{t}$, then $I^{\sigma}$ is a squarefree strongly stable ideal generated in degrees $d_{1}<d_{2}<\cdots<d_{t}$, as well. Similarly, if $I \subsetneq S$ is a squarefree strongly stable ideal generated in several degrees, then $I^{\tau}$ is a strongly stable ideal with generators in the same degrees as those of $I$. 
Theorem 5.5. Let $I \subsetneq S$ be a revlex ideal generated in degrees $d, d+1$. Then $I^{\sigma}$ is a squarefree revlex ideal of $K\left[x_{1}, \ldots, x_{m}\right]$, where $m=\max \{\max (u)+\operatorname{deg} u-1: u \in G(I)\}$.

Proof. Set $S^{\prime}=K\left[x_{1}, \ldots, x_{m}\right]$ with $m=\max \{\max (u)+\operatorname{deg} u-1$ : $u \in G(I)\}$. If $I$ is a revlex ideal in $S$ with $G(I)=\left\{u_{1}, \ldots, u_{s}\right\}$, then, from Lemma 5.2, one has that $I^{\sigma}$ is a squarefree strongly stable ideal of $S^{\prime}$ with $G\left(I^{\sigma}\right)=\left\{u_{1}^{\sigma}, \ldots, u_{s}^{\sigma}\right\}$.

Since $I$ is a revlex ideal in $S$, then the following condition is satisfied [12, Corollary 2.13]:

$$
x_{n-1}^{d+1} \in I .
$$

We claim that $x_{m-(d+2)} \cdots x_{m-3} x_{m-2} \in I^{\sigma}$.

Set $\widetilde{m}=\max \{\max (u): u \in G(I)\}$. From condition (2), one has $\widetilde{m} \in\{n-1, n\}$. Let $\widetilde{m}=n-1$. Then $m=n+d-1$ and, consequently, $m-(d+2)=n-3$. On the other hand, $x_{n-3}^{d+1}>_{\text {revlex }} x_{n-1}^{d+1}$. Therefore, since $I$ is a revlex ideal in $S$ and $x_{n-1}^{d+1} \in I$, it follows that $x_{n-3}^{d+1} \in I$. Since $\left(x_{n-3}^{d+1}\right)^{\sigma}=x_{n-3} x_{n-2} \cdots x_{n-3+d}=x_{m-(d+2)} \cdots x_{m-3} x_{m-2}$, the claim follows.

Let $\widetilde{m}=n$. Then $m=n+d$ and so $m-(d+2)=n-2$. One has that $x_{n-2}^{d+1}>_{\text {revlex }} x_{n-1}^{d+1}$. Therefore, since $x_{n-1}^{d+1} \in I$ and $I$ is a revlex ideal in $S$, it follows that $x_{n-2}^{d+1} \in I$. Finally, since $\left(x_{n-2}^{d+1}\right)^{\sigma}=x_{n-2} x_{n-1} \cdots x_{n-2+d}=x_{m-(d+2)} \cdots x_{m-3} x_{m-2}$, the claim follows.

Now we have to prove that $\operatorname{Mon}^{s}\left(I_{d}^{\sigma}\right)$ and $\operatorname{Mon}^{s}\left(I_{d+1}^{\sigma}\right)$ are squarefree revlex segments in $S^{\prime}$. Let $w \in I^{\sigma}$ be a squarefree monomial, and let $v>_{\text {srevlex }} w$ be a squarefree monomial of $S^{\prime}$ with $\operatorname{deg} v=\operatorname{deg} w$. We will show that $v \in I^{\sigma}$.

From (1), since $I^{\sigma}$ is a squarefree strongly stable ideal, one can decompose $w=u^{\sigma} y$, where $u \in G(I)$ and $y$ is a squarefree monomial with $\max \left(u^{\sigma}\right)<\min (y)$. Then $w^{\tau}=u z, z \in S$ and $w^{\tau} \in I$. Since $I$ is a revlex ideal, and since $v^{\tau}>_{\text {revlex }} w^{\tau}$, one obtains that $v^{\tau} \in I$. Hence, $v^{\tau}=u^{\prime} z^{\prime}$, where $u^{\prime} \in G(I)$ and $\max \left(u^{\prime}\right) \leq \min \left(z^{\prime}\right)$ [13, Lemma 1.1]. Therefore, $u^{\prime \sigma}$ divides $\left(v^{\tau}\right)^{\sigma}=v$. Hence, Corollary 3.3 ensures that $I^{\sigma}$ is a squarefree revlex ideal of $S^{\prime}$. 
Theorem 5.6. Let $I \subsetneq S$ be a squarefree revlex ideal generated in degrees $d, d+1$. Then $I^{\tau}$ is a revlex ideal of $K\left[x_{1}, \ldots, x_{m}\right]$, where $m=\max \{\max (u)-\operatorname{deg} u+1: u \in G(I)\}$.

Proof. If $I$ is a revlex ideal in $S$ with $G(I)=\left\{u_{1}, \ldots, u_{s}\right\}$, then, from Lemma 5.3, one has that $I^{\tau}$ is a squarefree strongly stable ideal of $K\left[x_{1}, \ldots, x_{m}\right], m=\max \{\max (u)-\operatorname{deg} u+1: u \in G(I)\}$, with $G\left(I^{\tau}\right)=\left\{u_{1}^{\tau}, \ldots, u_{s}^{\tau}\right\}$.

Since $I$ is a squarefree revlex ideal in $S$, then, from Corollary 3.3, the following condition is satisfied

$$
x_{n-(d+2)} \cdots x_{n-3} x_{n-2} \in I .
$$

We claim that $x_{m-1}^{d+1} \in I^{\tau}$.

Set $\widetilde{m}=\max \{\max (u): u \in G(I)\}$. From (3), one has $\widetilde{m} \in$ $\{n-2, n-1, n\}$.

Let $\tilde{m}=n-2$. Then $m=n-d-2$, and $m-1=n-(d+3)$. Consider the squarefree monomial $x_{n-(d+3)} x_{n-(d+2)} \cdots x_{n-3}$ of $S$. One has $x_{n-(d+3)} x_{n-(d+2)} \cdots x_{n-3}>_{\text {srevlex }} x_{n-(d+2)} \cdots x_{n-3} x_{n-2}$. Since $I$ is a squarefree revlex ideal of $S$, then $x_{n-(d+3)} x_{n-(d+2)} \cdots x_{n-3} \in I$. It follows that $\left(x_{n-(d+3)} x_{n-(d+2)} \cdots x_{n-3}\right)^{\tau}=x_{n-(d+3)}^{d+1}=x_{m-1}^{d+1} \in I^{\tau}$.

Let $\widetilde{m}=n-1$. Then $m=n-d-1$ and $m-1=n-(d+2)$. Since $x_{n-(d+2)} \cdots x_{n-2} \in I$, then $\left(x_{n-(d+2)} \cdots x_{n-2}\right)^{\tau}=x_{n-(d+2)}^{d+1}=x_{m-1}^{d+1} \in$ $I^{\tau}$.

Let $\widetilde{m}=n$. Then $m=n-d$ and $m-1=n-(d+1)$. Consider the squarefree monomial $x_{n-(d+1)} \cdots x_{n-1}$ of $S$. Let $u$ be the greatest monomial of $G(I)$ with respect to the revlex order such that $\max (u)=$ $n$. Since $x_{n-(d+1)} \cdots x_{n-1}>_{\text {srevlex }} u$, then $x_{n-(d+1)} \cdots x_{n-1} \in I$. It follows that $\left(x_{n-(d+1)} \cdots x_{n-1}\right)^{\tau}=x_{n-d-1}^{d+1}=x_{m-1}^{d+1} \in I^{\tau}$.

Checking that $\operatorname{Mon}\left(I_{d}^{\tau}\right)$ and $\operatorname{Mon}\left(I_{d+1}^{\tau}\right)$ are revlex segments in $S$ is similar to the proof given in Proposition 5.5 with the operator $\sigma$. The assertion follows from [12, Corollary 2.13].

Denote by $\boldsymbol{R} \boldsymbol{e} \boldsymbol{v}$ the class of all revlex ideals and by $\boldsymbol{R} \boldsymbol{e} \boldsymbol{v}^{\mathbf{s}}$ the class of all squarefree revlex ideals in $S$.

As a consequence of Proposition 3.4 and Theorem 5.6, we get the following corollary.

Corollary 5.7. There is an injection between $\boldsymbol{R e} \boldsymbol{v}^{s}$ and $\boldsymbol{R e v}$. 
6. Extremal Betti numbers of squarefree revlex ideals. In this section, we analyze the extremal Betti numbers of squarefree revlex ideals. Since the extremal Betti numbers of a squarefree monomial ideal $I$, as well as their positions, are preserved by passing to $I^{\tau}[\mathbf{3}$, Theorem 2.4], from Theorem 5.6, one can expect that a squarefree revlex ideal has a unique extremal Betti number as in the non-squarefree case $([8,10])$.

Definition 6.1. A Betti number $\beta_{k, k+\ell}(I) \neq 0$ is called extremal if $\beta_{i, i+j}(I)=0$ for all $i \geq k, j \geq \ell,(i, j) \neq(k, \ell)$.

The following results were proved in $[\mathbf{9}, \mathbf{1 1}]$.

Proposition 6.2. Let $I \subsetneq S$ be a squarefree stable ideal. The following conditions are equivalent:

(1) $\beta_{k, k+\ell}(I)$ is extremal;

(2) $k+\ell=\max \left\{\max (u): u \in G(I)_{\ell}\right\}$ and $\max (u)<k+j$ for all $j>\ell$ and $u \in G(I)_{j}$.

Corollary 6.3. Let $I \subsetneq S$ be a squarefree stable ideal.

(1) If $\beta_{k, k+\ell}(I)$ is an extremal Betti number of $I$, then

$$
\beta_{k, k+\ell}(I)=\left|\left\{u \in G(I)_{\ell}: \max (u)=k+\ell\right\}\right| .
$$

(2) Set $d=\max \left\{j: G(I)_{j} \neq \emptyset\right\}$ and $m=\max \{\max (u): u \in G(I)\}$. Then $\beta_{m-d, m}(I)$ is the unique extremal Betti number of $I$ if and only if $m=\max \left\{\max (u): u \in G(I)_{d}\right\}$ and for every $w \in G(I)_{j}$, $j<d, \max (w)<m$.

Proposition 6.4. Let $I \subsetneq S$ be a squarefree revlex ideal. Then $I$ has a unique extremal Betti number.

Proof. The assertion follows from Proposition 3.4 and Corollary 6.3.

In fact, if $I$ is generated in degree $d$, then $\beta_{k, k+d}(I)$ is the unique extremal Betti number of $I$ with $k+d=\max \left\{\max (u): u \in G(I)_{d}\right\} \in$ $\{n-2, n-1, n\}$.

Let $I$ be generated in degrees $d, d+1$. If $x_{n-(d+1)} \cdots x_{n-2} \in$ $G(I)_{d}$, then $\beta_{k, k+d+1}(I)$ is the unique extremal Betti number of $I$ with 
$k+d+1=\max \left\{\max (u): u \in G(I)_{d+1}\right\}=n$. If $x_{n-(d+2)} \cdots x_{n-2} \in$ $G(I)_{d+1}$, then $\beta_{k, k+d+1}(I)$ is the unique extremal Betti number of $I$ with $k+d+1=\max \left\{\max (u): u \in G(I)_{d+1}\right\} \in\{n-2, n-1, n\}$.

Remark 6.5. Let $I \subsetneq S$ be a squarefree revlex ideal with extremal Betti number $\beta_{k, k+\ell}(I)$. Then, from Corollary 6.3,

$$
1 \leq \beta_{k, k+\ell}(I) \leq\left(\begin{array}{c}
k+\ell-1 \\
\ell-1
\end{array}\right)
$$

In fact, there are exactly $\left(\begin{array}{c}k+\ell-1 \\ \ell-1\end{array}\right)$ squarefree monomials of degree $\ell$ in $S$ with $\max (u)=k+\ell$.

More precisely, let $I$ be generated in degree $\ell$. Set $k+\ell=n-2+i$, for $i=0,1,2$. Then

$$
1 \leq \beta_{k, k+\ell}(I) \leq\left(\begin{array}{c}
n-3+i \\
\ell-1
\end{array}\right) .
$$

Let $I$ be generated in degrees $d$ and $d+1$. Therefore, $\ell=d+1$.

Suppose $x_{n-\ell} \cdots x_{n-2} \in G(I)_{\ell-1}$. Set $k+\ell=n$. Then

$$
1 \leq \beta_{k, k+\ell}(I) \leq\left(\begin{array}{c}
n-1 \\
\ell-1
\end{array}\right) .
$$

Suppose $x_{n-(\ell+1)} \cdots x_{n-2} \in G(I)_{\ell}$. Set $k+\ell=n-2+i$, for $i=0,1,2$. Then

$$
1 \leq \beta_{k, k+\ell}(I) \leq\left(\begin{array}{c}
n-3+i \\
\ell-1
\end{array}\right)
$$

For $u, v \in \operatorname{Mon}_{d}^{s}(S)$ with $u>$ srevlex $v$, we define the following set:

$$
\mathfrak{R}(u, v):=\left\{w \in \operatorname{Mon}_{d}^{s}(S): u \geq_{\text {srevlex }} w \geq_{\text {srevlex }} v\right\} .
$$

Finally, we have the following result.

Proposition 6.6. Given a pair of positive integers $(k, \ell)$ such that $k \geq$ 1 and $\ell \geq 2$, then there exists a squarefree revlex ideal $I \subsetneq K\left[x_{1}, \ldots, x_{n}\right]$ with extremal Betti number $\beta_{k, k+\ell}(I)=a$ if and only if one of the following conditions holds: 
(i) $k+\ell=n-2$ and $1 \leq a \leq\left(\begin{array}{c}n-3 \\ \ell-1\end{array}\right)$;

(ii) $k+\ell=n-1$ and $1 \leq a \leq\left(\begin{array}{c}n-2 \\ \ell-1\end{array}\right)$;

(iii) $k+\ell=n$ and $1 \leq a \leq\left(\begin{array}{c}n-1 \\ \ell-1\end{array}\right)$.

Proof. Suppose $I$ is a squarefree revlex ideal with extremal Betti number $\beta_{k, k+\ell}(I)=a$. Then conditions (i), (ii) and (iii) follow from Remark 6.5.

Let us now suppose that condition (i) holds. If $a=\left(\begin{array}{c}n-3 \\ \ell-1\end{array}\right)$, we construct the squarefree revlex ideal $I$ of $S$ generated in degree $\ell$ with $G(I)=\mathfrak{R}\left(x_{1} \cdots x_{\ell}, x_{n-(\ell+1)} \cdots x_{n-2}\right)$. Suppose $1 \leq a<\left(\begin{array}{c}n-3 \\ \ell-1\end{array}\right)$. Let $W=\left\{u \in \operatorname{Mon}_{\ell}^{s}(S): \max (u)=n-2\right\}$. Set $W=\left\{w_{1}, w_{2}, \ldots, w_{r}\right\}$, where $w_{1}>_{\text {srevlex }} w_{2}>_{\text {srevlex }} \cdots>_{\text {srevlex }} w_{r}=x_{n-(\ell+1)} \cdots x_{n-2}$. We construct the squarefree revlex ideal $I$ of $S$ generated in degrees $\ell-1$ and $\ell$ with $G(I)=A \cup B$, where $A$ is given by all monomials of degree $\ell-1$ from $x_{1} \cdots x_{\ell-1}$ down to the greatest monomial $z \in \operatorname{Mon}_{\ell-1}^{s}(S)$, with respect to the squarefree revlex order, such that $z x_{n-2}=w_{r-a}$, and $B=\mathfrak{R}\left(w_{r-a+1}, w_{r}\right)$. Note that $|B|=a$.

Suppose condition (ii) holds. We construct the squarefree revlex ideal $I$ of $S$ generated in degree $\ell$ with $G(I)=\mathfrak{R}\left(x_{1} \cdots x_{\ell}, x_{n-(\ell+1)} \cdots x_{n-2}\right)$ $\cup A$, where $A$ consists of the largest $a$ monomials $u$ less than $x_{n-(\ell+1)} \cdots$ $x_{n-2}$ with respect to the squarefree revlex order on $S$ such that $\max (u)=n-1$.

Suppose condition (iii) holds. We construct the squarefree revlex ideal $I$ of $S$ generated in degree $\ell$ with $G(I)=\mathfrak{R}\left(x_{1} \cdots x_{\ell}, x_{n-\ell} \cdots x_{n-1}\right)$ $\cup A$, where $A$ consists of the largest $a$ monomials $u$ less than $x_{n-\ell} \cdots$ $x_{n-1}$ with respect to the squarefree revlex order such that $\max (u)=$ $n$.

Acknowledgments. We thank the anonymous referee for his/her careful reading and useful comments that improved the quality of the paper.

\section{REFERENCES}

1. A. Aramova, J. Herzog and T. Hibi, Gotzmann theorems for exterior algebras and combinatorics, J. Algebra 191 (1997) 174-211.

2. Squarefree lexsegment ideals, Math. Z. 228 (1998) 353-378. 
3. A. Aramova, J. Herzog and T. Hibi, Shifting operations and graded Betti numbers, J. Alg. Comb. 12 (2000), 207-222.

4. A. Bigatti, Upper bounds for the Betti numbers of a given Hilbert function, Comm. Algebra21 (1993), 2317-2334.

5. H. Brenner, J. Herzog and O. Villamayor, Three lectures on Commutative algebra, University Lect. Ser. 42, American Mathematical Society-RSME, 2008.

6. M. Crupi and C. Ferrò, Hilbert functions and Betti numbers of reverse lexicographic ideals in the exterior algebra, Turkish J. Math. 36 (2012), 366-375.

7. , Revlex ideals in the exterior algebra, Math. Rep. 15 (2013), 193-201.

8. M. Crupi and M. La Barbiera, Minimal graded resolutions of reverse lexsegment ideals, Math. Notes 91 (2012), 364-377.

9. _ Algebraic properties of universal squarefree lexsegment ideals, Algebra Coll. (accepted for publication in 2013); preprint available at arXiv: $1409.8026 \mathrm{v} 1$.

10. M. Crupi and R. Utano, Extremal Betti numbers of lexsegment ideals, Lect. Notes Pure Appl. Math. 217 (2000), 159-164.

11. _ Extremal Betti numbers of graded ideals, Results Math. 43 (2003), $235-244$.

12. T. Deery, Revlex segment ideals and minimal Betti numbers, The Curves Seminar at Queen's, vol. X: Queen's Papers Pure Appl. Math. 102, Queen's University Press, Kingston, ON, 1996.

13. S. Eliahou and M. Kervaire, Minimal resolutions of some monomial ideals, J. Algebra 129 (1990), 1-25.

14. J. Herzog and T. Hibi, Monomial ideals, Grad. Texts Math., Springer, New York, 2010.

15. H. Hulett, Maximum Betti numbers for a given Hilbert function, Comm. Algebra 21 (1993), 2335-2350.

16. K. Pardue, Deformation classes of graded modules and maximal Betti numbers, Illinois J. Math. 40 (1996), 564-585.

17. G.A. Reisner, Cohen-Macaulay quotients of polynomial rings, Adv. Math. 21 (1976), 30-49.

18. R.P. Stanley, The upper bound conjecture and Cohen-Macaulay rings, Stud. Appl. Math. 54 (1975), 135-142.

Department of Mathematics and Computer Science, University of Messina, Viale F. Stagno d'Alcontres, 31, 98166 Messina, Italy

Email address: mcrupi@unime.it

Department of Mathematics and Computer Science, University of Messina, Viale F. Stagno d'Alcontres, 31, 98166 Messina, Italy

Email address: cferro@unime.it 